early in development, neurons exhibit spontaneous electrical activity that affects the process of neuronal specialization and the establishment of appropriate connections ${ }^{7.8}$. Changes in activity during development can indeed lead to resetting of the intrinsic excitability of neurons as well as reconfiguration of connections'. In particular, formation of motor central pattern generators requires early spontaneous electrical activity, because when this activity is disrupted, motor neuron fibres fail to follow their normal trajectories ${ }^{10}$.

However, Gosgnach et al. ${ }^{4}$ find that the activity of V1 neurons does not seem to be involved in the formation of the central pattern generator. Their results show that loss of V1 neurons early in development is not compensated for by any reconfiguration of the circuit - the mutant mice that chronically lost V1 neurons at early stages exhibit the same slow locomotor rhythm as that observed when neurons are acutely silenced after the circuit is formed.

Nevertheless, studies of rewiring during development are complex; they require a rather dynamic approach because exclusive examination of the final end point may not reveal intermediate remodelling processes that are crucial to the resulting network. In this regard, it would be interesting to test what happens to the circuit and locomotor activity if neurons are silenced at different times during development. Formation of circuits is likely to depend on both genetically driven and activity-shaped processes. We need to keep our minds open if we are to understand the interplay of these driving forces.

Developmental biologists and physiologists have tended to approach the problem of circuit formation and function from very different angles, without much dialogue between them. But genetic tools are now available to reconcile the results from these two disciplines and establish a fruitful interaction. Information about signatures of gene-regulatory factors that determine the fates of neuronal populations can now serve as tools with which to elucidate the functions of a given set of neurons. The work presented by Gosgnach et al. sets up a firm bridge across the river that has so far divided developmental biology and physiology.

Laura N. Borodinsky is in the Neurobiology

Section, Division of Biological Sciences, University of California, San Diego, 9500 Gilman Drive, La Jolla, California 92093-0357, USA. e-mail: Iborodin@biomail.ucsd.edu

1. Sherrington, C. J.Physiol. (Lond) 47,196-214 (1913).

2. Hamburger, $V_{\gamma}$ Wenger, E.\& Oppenheim, R.J. Exp Zool. $162,133-160$ (1966).

3 Brown T. Proc. R. Soc. Land B 84, 308-319 (1911).

4. Gosgnach, S.etal. Nature 440,215-219 (2006).

5 Lanuza, G.M. Gosgnach, S, Plerani, A, lessell, T.M.\& Goulding M.Neuran 42, 375-386 (2004).

6. Tanabe, Y \& lessell, T. M. Science 274,1115-1123(1996).

7. Gu X.\& Spitzer, N.C. Nature375, 784-787(1995)

8. Catalano, S.M. \& Shatz, C.J.Science 281, 559-562 (1998),

9. Turrigiano, G.G. \& Nelson, S.B. Nature Rev. Neurasci.5, 97-107(2004).

10. Hanson, M. G \& Landmesser, L. T. Neuran 43, 687-701 (2004).

\title{
BIOENGINEERING
}

\section{Diagnosis on disc}

\author{
Frances S. Ligler and Jeffrey S. Erickson
}

\section{Highly complex immunoassays that identify and quantify many different antigens simultaneously need high-resolution imaging capability. A simple, low-cost technique could be music to our ears.}

The capabilities of immunoassays - tests that use the binding of 'capture' antibodies to antigens in order to identify the latter - have been advancing in leaps and bounds over the past three decades. Methods of enzyme amplification have increased assay sensitivity. Diode lasers have reduced the size and cost of instrumentation. Microfluidics has enabled both the analysis of very small sample volumes and the parallel processing of multiple samples. And microarrays of capture antibodies, or other recognition molecules, attached to surfaces have made possible the simultaneous testing of a sample for large numbers of target molecules.

This last point harbours a problem. As microarray elements become smaller and smaller for larger and larger numbers of simultaneous tests, the necessary imaging resolution cannot readily be achieved with the standard, off-the-shelf CMOS or CCD technologies. Confocal scanners using microscope lenses can provide the required resolution, but their cost, size and the geometrical alignment of their optics pose other practical problems. Writing in Angewandte Chemie International Edition, Sebastian A. Lange, Günter Roth and colleagues find a way out of this seeming impasse ${ }^{1}$. They demonstrate an immunoassay readout of high efficiency and sensitivity using, in a slightly modified form, a pick-up head of a compact-disc player (Fig. 1).

The authors' system is both elegant and simple. They used a so-called sandwich immunoassay, in which the antigen to be measured is bound between two different antibodies. First, a capture antibody is stamped in a 25-micrometre square pattern onto a solid substrate; the desired antigen binds to this antibody. Second, a detector antibody binds to this antigen to make it visible to the $\mathrm{CD}$ pickup head. Here, the authors cleverly employed an antibody carrying a gold nanoparticle. This gold nanoparticle catalyses the deposition of silver grains onto the substrate to which the capture antibody, antigen and detector antibody are all now attached. The reflections from the silver grains could be read by the $C D$ pick-up head with a resolution of around 50 nanometres - considerably smaller than the diameter of most of the silver nanoparticles, which is of the order of several hundred nanometres. Using their sandwich system, the authors demonstrated that they could detect antigen in serum at concentrations from 1 microgram per millilitre down to 100 picograms per millilitre.

The authors also performed a carefully controlled study in which they spaced antigens, each attached to a capture molecule, well apart from each other on the substrate ${ }^{1}$. They suggest that, by correlating the density of the silver precipitate with the coverage of the antigen, it might be possible to detect single molecules with their system. But measuring a single molecule carefully tied to a uniform surface is a far cry from pulling a single molecule out of a solution, capturing it at a surface and measuring it against a background signal generated from any of several variable sources. The potential for using the $C D$ pick-up head with silver staining for single-molecule detection is therefore less convincing than the suggestion that the technology could be widely useful for low-cost, high-sensitivity readout
Figure 1|Molecularmusic. A conventional compact-disc pick-up reader works by focusing laser light onto the surface of the CD. As the CD rotates above the reader, information encoded as pits along a spiral track on its metal-coated surface can be read by means of light reflected back through a lens onto a photodiode. Lange and colleagues' immunoassay system ${ }^{1}$ works in exactly the same way, but with a substrate covered with a regularly spaced array of capture antibodies taking the place of the CD. When an antigen binds to an antibody, its presence is signalled by a second, gold-containing detector antibody that catalyses the deposition of reflective silver particles onto the substrate.

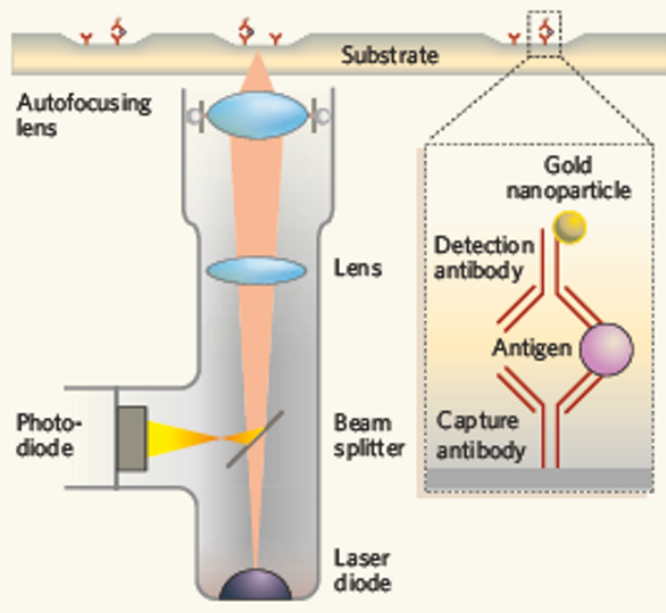


systems for many different types of assay.

Significant issues remain to be addressed. If the CD pick-up head is to be used to detect many different molecules simultaneously, multiple capture molecules will have to be immobilized on surfaces suitable for use with the CD pick-up heads. This is almost sure to require a more flexible technology than the stamping method used by Lange et al. Optical alignment issues will also need to be addressed. In Lange and colleagues' study, the CD pick-up head is only one part of the optics: it is combined with a microscope stage for coarse adjustment of the magnification, as well as a lateral translation stage to adjust the position of the line of sight over the substrate. Although it may be possible to use built-in hardware in a commercially available CD player to perform these functions, this has yet to be demonstrated.

One noteworthy advantage of the CD-pickup approach, alongside small size, low cost and high resolution, is not emphasized by the authors. Although an assay signal has never actually been generated using a $C D$ as a sensing surface, biochemical manipulations have already been performed directly on a CD.
Externally readable fluorescence signals have even been generated in channels within the disc $^{2-5}$ - the challenge here being the use of centrifugal force to move the fluids through the processing steps on the surface of the CD to the readout position. The combination of such fluidic approaches with in situ signal generation as demonstrated by Lange et al. ${ }^{1}$ could potentially lead to a sea change in medical diagnostics. Imagine in the future buying a 'respiratory pathogen $C D$ ' from the local pharmacy when you catch a cold, inserting your self-test swab and placing it in your portable player to find out whether you should take antibiotics or stick to the chicken soup. [ Frances S. Ligler and Jeffrey S. Erickson are in the Center for Bio/Molecular Science and Engineering Naval Research Laboratory, Code 6900, Washington DC 20375-5348, USA. e-mails: fligler@cbmse.nrl.navy.mil; jerickson@cbmse.nrl.navy.mil

1. Lange, S.A.et al. Angew. Chem. Int. Edn 45, 270-273(2006) 2. Rothert, A. et al. Anal Biochem. 342, 11-19 (2005).

3. Gustafsson, M, Hirschberg $D_{7}$ Palmberg $C$, Jornvall, $H$ \& \& Bergman T. Anal Chem. 76, 345-350 (2004).

4. Tibbe, A.G Let al. Cytometry 47,173-182(2002)

5. Lai, S.etal. Anal.Chem. 76, 1832-1837 (2004).

\title{
PHYSICAL CHEMISTRY
}

\section{Stressed molecules break down}

\author{
Steve Granick and Sung Chul Bae
}

\section{Tough carbon-carbon bonds can snap in certain large molecules just because the two sides of the molecule cannot agree on which way to go during adsorption. Heresy? The view through the microscope suggests otherwise.}

On page 191 of this issue ${ }^{1}$, Sheiko and colleagues show that the mechanical deformation induced merely by the adhesion of a complex molecule to a surface can trigger the break-up of that molecule. They thus provide convincing support for the seemingly heretical notion that the commonplace and unremarkable process of adsorption to a surface can bring about what otherwise occurs only with the greatest effort: the rupture of the strong, covalent carbon-carbon bond.

The system designed by the authors ${ }^{1}$ is elegant in its simplicity. They placed brush-like, polymeric macromolecules on various solid and liquid surfaces to which the molecules' side-chains (the 'bristles') were strongly attracted. This attraction drove the bristles to spread out so as to maximize their contact with the surface, in turn causing the polymeric backbone of the molecule to stretch until it was eventually strained too far. Direct imaging of the size of the molecules using atomic force microscopy proved that they had been torn apart, just as if the rope had failed in a game of tug of war (Fig. 1).

When a chemical bond snaps, a chemical reaction takes place. But exactly how can a purely mechanical effect have chemical consequences? That is the wider question investigated by a field known as chemomechanics? Future research in this area might well focus on whether mechanical stress shifts the energy level of the transition state of a chemical
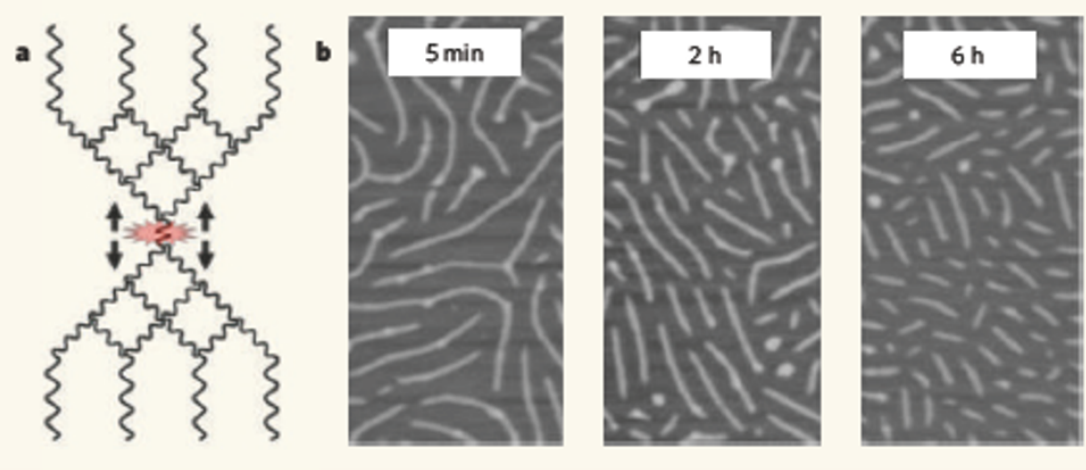

Figure 1 | Bond breaking. a, Channelling mechanical stress to specific, weaker chemical bonds can trigger chemical reactions that otherwise occur only with great effort. $b$, Sheiko et al. ${ }^{1}$ implemented this idea with brush-like macromolecules on a solid surface. As the bristles of these brushes spread to maximize their contact with the surface, the resultant force is concentrated at the middle, causing chemical bonds to break: the molecules' length thus decreases with time. 